\title{
Case Report: Response to Almonertinib in a Patient With Metastatic NSCLC Resistant to Osimertinib due to Acquired EGFR L718Q Mutation
}

\author{
Gang Shen ${ }^{1+}$, Lei Shi ${ }^{1+}$, Xin Tian ${ }^{2}$, Depei Huang ${ }^{3}$, Hao Chen ${ }^{4}$, Chan Gao ${ }^{3}$, Xudong Shen ${ }^{3}$ and \\ Hushan Zhang ${ }^{3 *}$ \\ ${ }^{1}$ Department of Thoracic Oncology, The Second Affiliated Hospital of Zunyi Medical University, Zunyi, China, ${ }^{2}$ Department of \\ Head and Neck Oncology, The Second Affiliated Hospital of Zunyi Medical University, Zunyi, China, ${ }^{3}$ The Medical Department, 3D \\ Medicines Inc., Shanghai, China, ${ }^{4}$ The Bioinformatics Department, 3D Medicines Inc., Shanghai, China
}

\section{OPEN ACCESS}

Edited by: Monica Montopoli, University of Padua, Italy

Reviewed by: Teodora Alexa-Stratulat, Grigore T. Popa University of Medicine and Pharmacy, Romania Hao Liu,

Bengbu Medical College, China

*Correspondence: Hushan Zhang

15111010041@fudan.edu.cn

${ }^{t}$ These authors have contributed equally to this work

Specialty section:

This article was submitted to Pharmacology of Anti-Cancer Drugs,

a section of the journal

Frontiers in Pharmacology

Received: 20 July 2021 Accepted: 29 November 2021 Published: 20 December 2021

Citation:

Shen G, Shi L, Tian X, Huang D, Chen $H$, Gao $C$, Shen $X$ and Zhang $H$ (2021) Case Report: Response to

Almonertinib in a Patient With

Metastatic NSCLC Resistant to Osimertinib due to Acquired EGFR L718Q Mutation.

Front. Pharmacol. 12:731895. doi: 10.3389/fphar.2021.731895
Osimertinib shows strong clinical activity in first- and second-line treatment of nonsmall-cell lung cancer (NSCLC) patients with epidermal growth factor receptor (EGFR) mutations, especially EGFR T790M. However, when patients develop resistance, there is currently no definite postosimertinib treatment option. Herein, we report a patient with metastatic NSCLC who benefited from almonertinib after developing resistance to osimertinib.

Keywords: NSCLC-lung adenocarcinoma-EGFR-ALK-BRAF-KRAS-RET-MET-PD-L1-ROS1., EGFR, TKI-tyrosine kinase inhibitor, almonertinib, $\mathrm{L7180}$

\section{INTRODUCTION}

EGFR is one of the four HER family receptor members, and the EGFR gene is an important oncogene in NSCLC. Blocking EGFR by specific TKIs through competitive binding with the EGFR binding region can inhibit EGFR activation and its downstream signaling pathways, with significant antitumor effects in NSCLC. EGFR TKIs such as gefitinib, erlotinib, afatinib, icotinib and thirdgeneration TKIs such as osimertinib have been approved as standard care for NSCLC patients with mutations in the EGFR tyrosine kinase domain (TKD). However, acquired resistance almost inevitably develops after $9-15$ months of treatment. Several mechanisms of acquired resistance have been demonstrated, including secondary EGFR mutations and mutations in bypass signaling pathways (Liu et al., 2017; Wu and Shih, 2018). The T790M mutation, involving substitution of threonine to methionine at amino acid 790, is the most common mechanism of resistance to firstand second-line EGFR TKI treatment. Osimertinib, currently approved as standard first-line treatment for NSCLC patients carrying EGFR mutations, also shows good activity for NSCLC patients with EGFR T790M mutants (Remon et al., 2018). Unfortunately, these patients, even those with EGFR T790M mutations, also eventually develop acquired resistance to osimertinib. The mechanisms reported include activating mutations in the bypass signaling pathway, loss of the T790M mutation, histological transformation, and acquisition of the EGFR C797S or EGFR L718Q mutation (Ma et al., 2019; Taniguchi et al., 2019; Mu et al., 2020). Overcoming drug resistance is a

Abbreviations: EGFR, Epidermal Growth Factor Receptor; NGS, next-generation sequencing; NSCLC, Non-small cell lung cancer. 

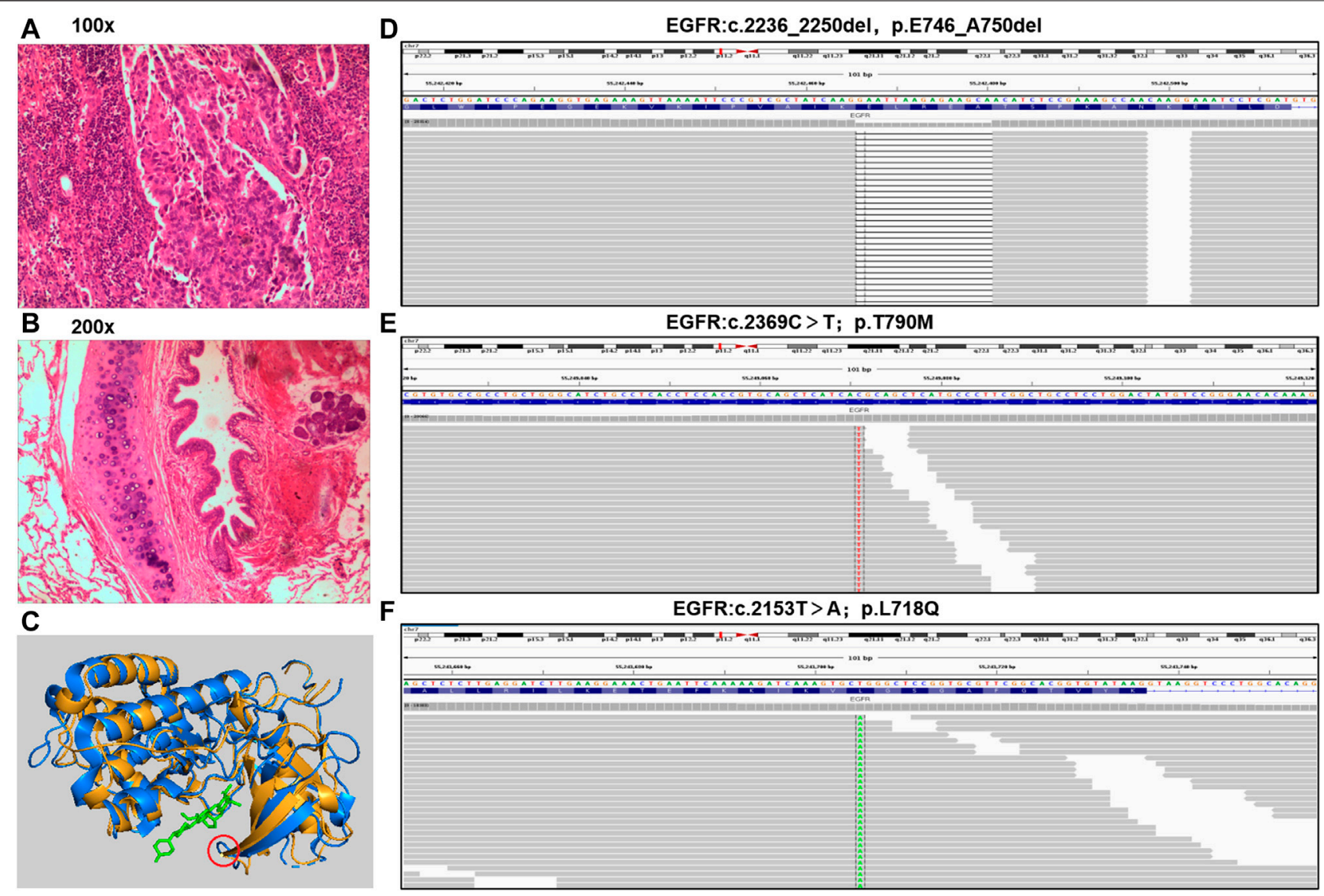

FIGURE 1 | Pathological diagnosis and molecular diagnosis. (A,B), tumor specimen was applied to apex of right lung with a pathological diagnosis of lung adenocarcinoma (hematoxylin-eosin staining: 100x and 200x). (C), Three-dimensional structure of EGFR protein with L718Q mutation predicted by MODELLER. Yellow represent structure of EGFR T790M, blue represent EGFR L718Q mutation, green represent TKI, and red circle present site of L718Q mutation. (D-F), the mutations of EGFR were analyzed through NGS in a laboratory accredited by the College of American Pathologists (CAP) and Clinical Laboratory Improvement Amendment (CLIA) (3D Medicines Inc., Shanghai, China).

clinical problem that needs to be solved urgently, but there is no clear and solid strategy to date. Some alternatives for overcoming resistance to osimertinib have recently been reported, such as osimertinib, bevacizumab, and brigatinib combination therapy and brigatinib (Uchibori et al., 2017; Zhao et al., 2018; Shaikh et al., 2021). Based on a phase 2 expansion study of a phase $1 / 2$ trial, the third-generation EGFR inhibitor almonertinib was approved in China in March 2020 for the treatment of advanced EGFR T790M-positive NSCLC (Yang et al., 2020; Nagasaka et al., 2021). Recent studies report that almonertinib has a good safety profile, with the parent drug as its main circulating component, and pharmacokinetic studies in a mouse model demonstrate a good ability of almonertinib to cross the blood-brain barrier (Zhang et al., 2021; Zhou et al., 2021). Herein, we present a case of resistance to osimertinib due to acquired EGFR L718Q mutation and subsequent response to almonertinib.

\section{Case Presentation}

A 45-year-old Chinese woman was admitted to the Affiliated Hospital of Zunyi Medical University for comprehensive treatment of metastatic NSCLC. She underwent thoracoscopic right middle and lower lobectomy, partial left atrial resection, pericardiotomy, and lymph node dissection on November 21, 2017, with a confirmed histopathological diagnosis of stage IIIC adenocarcinoma of the right middle lobe (pT4N3M0) (Figures 1A,B). Postoperative diagnosis showed no tumor involvement in the parenchyma of the right lower lobe but cancer infiltration in the left atrial wall and left parabronchial bronchi; metastasis was detected in some mediastinal lymph nodes. The patient was found to carry an EGFR exon 19 deletion mutation by analysis of tissue specimens using next-generation sequencing (NGS) (Figure 1C). She received icotinib $(125 \mathrm{mg} / \mathrm{d}$, tid) treatment (Figure 2A). Eight months after icotinib treatment, CT showed metastases in the liver. Previous studies have demonstrated that patients develop resistance after a median of 10 months of EGFR TKI treatment (Wu and Shih, 2018). Based on this situation, icotinib was discontinued. Chemotherapy was performed for 24 days, and computed tomography (CT) revealed thoracic spine metastasis, suggestive of prior treatment failure. She then began to receive icotinib combined with bevacizumab, which was switched to chemotherapy 6 months later due to severe 


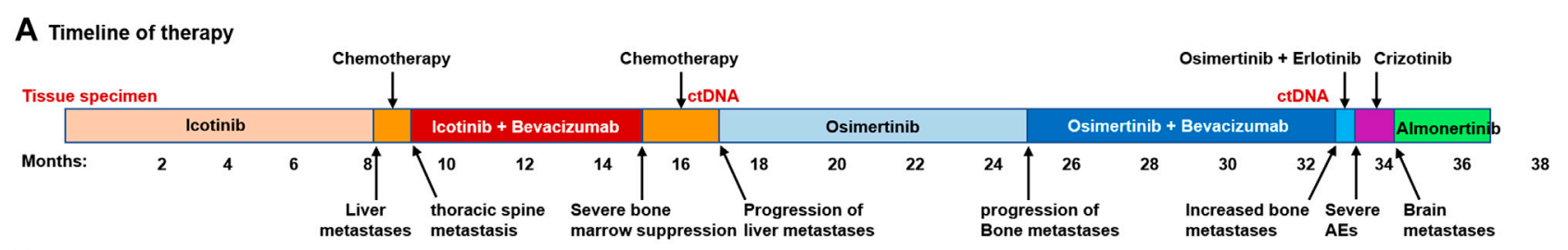

B Effect of therapy

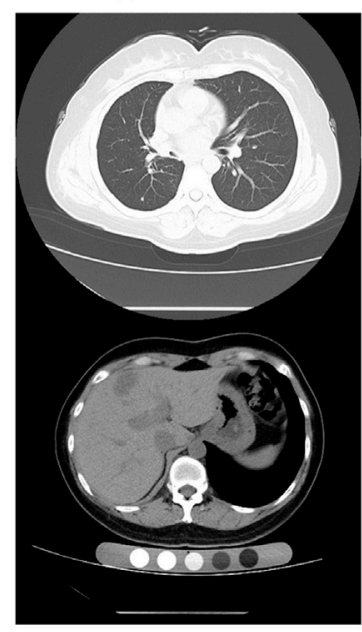

Before osimertinib

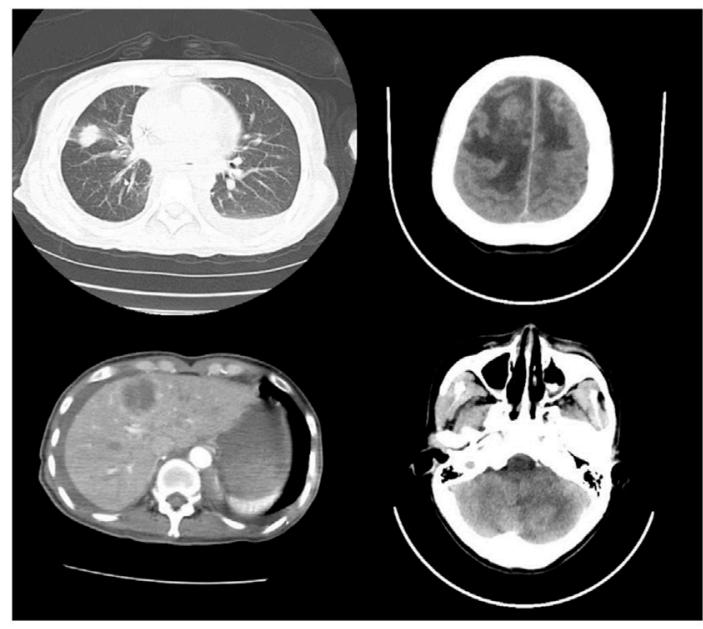

Resistance osimertinib

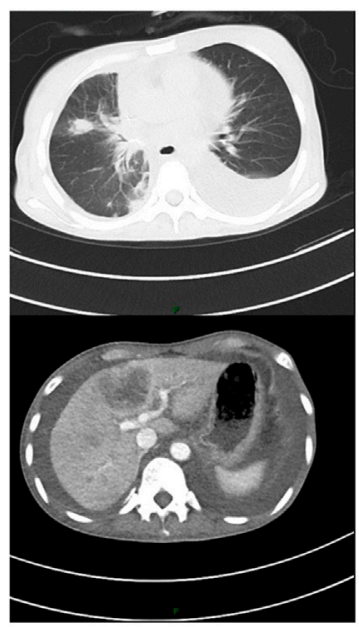

Response to almonertinib

FIGURE 2 | Acquired resistance to osimertinib and response to almonertinib. (A), the various treatments the patient received and the duration of the various treatments. (B), images of the patient's metastatic lung, liver and brain disease.

bone marrow suppression. A CT scan at that time showed progressive disease (PD), and analysis of circulating tumor DNA (ctDNA) revealed the EGFR T790M mutation (Figure 2B, 1D). She received osimertinib ( $80 \mathrm{mg}$, orally once daily) for 8 months, and then bevacizumab was added due to the progression of bone metastases. Eight months later, she was again found to have PD. Analysis of ctDNA showed the occurrence of EGFR L718Q (Figure 1E) and PLEKHH2-ALK fusions in addition to EGFR T790M. Therefore, osimertinib combined with erlotinib (150 mg, orally once daily) was initiated. Two weeks later, treatment was switched to crizotinib (250 mg twice daily) due to adverse events. One month later, the patient experienced dizziness, headache, and apathy. CT showed metastases in the bilateral parietal and left cerebellar regions and enlargement of the metastases in the liver (Figure 2B). Crizotinib was discontinued. The patient could not tolerate brain radiotherapy and thus was given almonertinib (110 mg, orally once daily), and she showed a clinical response to almonertinib that lasted more than 2 months. Overall, her symptoms of dizziness, headache relieved, and mental state improved significantly at 1 week after almonertinib was initiated, and her Karnofsky score increased from 20 to 70 . A CT scan two and a half months after almonertinib treatment initiation showed that both the liver and lung metastases were stable (Figure 2B). Four months after almonertinib therapy, she was discharged from the hospital, though almonertinib was discontinued because of serious electrolyte disorders and heart failure.

\section{DISCUSSION}

Despite the robust efficacy of osimertinib, resistance to this drug is inevitable due to the lack of postosimertinib options (Leonetti et al., 2019). Mutation at EGFR L718 are responsible for osimertinib resistance in vitro and in vivo, and the amino acid is most frequently substituted with glutamine. This has recently been shown to be a mechanism of resistance to osimertinib, but few therapies can effectively overcome it (Yang et al., 2018; Leonetti et al., 2019). In the crystal structure of osimertinib in complex with EGFR, L718 is in direct contact with the aniline ring. Thus, the L718Q mutation may mediate drug resistance through steric hindrance and influence drug binding; that is, the glutamine side chain at position 718 is expected to sterically interfere with the position of osimertinib, reducing the efficiency of covalent bond formation (Yosaatmadja et al., 2015; Bersanelli et al., 2016). We used MODELLER, a computational protein structure modeling (Webb and Sali, 2016) technique, to predict the three-dimensional structure of the EGFR protein with the L718Q mutation, and the predicted structure is supported by the above theory that mutation of EGFR L718Q may affect binding between osimertinib and EGFR-TKD (Figure 1F). Amino acid 718 is found inside the p-loop of the EGFR TKD, which interacts with the aniline ring of osimertinib. According to the predicted model, mutation of this residue may cause spatial restriction and prevent osimertinib from binding to EGFR, that is, cause resistance to the drug. Despite some case reports that patients carrying the EGFR L718Q mutation might 
respond to afatinib (Starrett et al., 2020), there are no feasible and solid strategies available for overcoming acquired resistance to osimertinib caused by EGFR L718Q, especially with EGFR T790M and L718Q comutation (Shaikh et al., 2021). In fact, previous case reports and in vitro studies have revealed that afatinib only exhibits an inhibitory effect on phosphorylation in the presence of EGFR L858R + L718V/Q comutation (Starrett et al., 2020). Although our case involved EGFR L718Q in addition to the T790M mutation, resistance to osimertinib had already emerged.

The safety and efficacy of almonertinib (HS-10296), a novel, thirdgeneration epidermal growth factor receptor tyrosine kinase inhibitor (EGFR-TKI), was evaluated in a phase I trial including patients with locally advanced or metastatic EGFR-mutated nonsmall-cell lung cancer (NSCLC) whose disease had progressed after prior EGFR TKI therapy (Yang et al., 2020). As previously reported, the main adverse events of all grades with almonertinib treatment of NSCLC patients were rash, pruritis, leukopenia, and increases in creatinine phosphokinase, alanine aminotransferase and aspartate aminotransferase, with increases in creatinine phosphokinase and alanine aminotransferase being the most common grade 3-4 adverse events (Yang et al., 2020; Nagasaka et al., 2021). In our case, unfortunately, almonertinib treatment ultimately discontinued due to serious electrolyte disorders and heart failure. However, according to previous evidence, electrolyte disorders and heart failure may be associated with accumulation of toxicity due to previous multiline treatment, whereby the physical constitution becomes very poor, rather than being due to almonertinib treatment.

\section{CONCLUSION}

We report an NSCLC patient with metastasis and osimertinib resistance who benefitted from almonertinib. First, this case confirms resistance to osimertinib caused by the L718Q

\section{REFERENCES}

Bersanelli, M., Minari, R., Bordi, P., Gnetti, L., Bozzetti, C., Squadrilli, A., et al. (2016). L718Q Mutation as New Mechanism of Acquired Resistance to AZD9291 in EGFR-Mutated NSCLC. J. Thorac. Oncol. 11 (10), e121-3. doi:10.1016/j.jtho.2016.05.019

Leonetti, A., Sharma, S., Minari, R., Perego, P., Giovannetti, E., and Tiseo, M. (2019). Resistance Mechanisms to Osimertinib in EGFR-Mutated Non-small Cell Lung Cancer. Br. J. Cancer 121 (9), 725-737. doi:10.1038/s41416-0190573-8

Liu, X., Wang, P., Zhang, C., and Ma, Z. (2017). Epidermal Growth Factor Receptor (EGFR): A Rising star in the Era of Precision Medicine of Lung Cancer. Oncotarget 8 (30), 50209-50220. doi:10.18632/oncotarget.16854

Ma, L., Chen, R., Wang, F., Ma, L. L., Yuan, M. M., Chen, R. R., et al. (2019). EGFR L718Q Mutation Occurs without T790M Mutation in a Lung Adenocarcinoma Patient with Acquired Resistance to Osimertinib. Ann. Transl Med. 7 (9), 207. doi:10.21037/atm.2019.04.37

Mu, Y., Hao, X., Xing, P., Hu, X., Wang, Y., Li, T., et al. (2020). Acquired Resistance to Osimertinib in Patients with Non-small-cell Lung Cancer: Mechanisms and Clinical Outcomes. J. Cancer Res. Clin. Oncol. 146 (9), 2427-2433. doi:10.1007/ s00432-020-03239-1

Nagasaka, M., Zhu, V. W., Lim, S. M., Greco, M., Wu, F., and Ou, S. I. (2021). Beyond Osimertinib: The Development of Third-Generation EGFR Tyrosine mutation. In addition, this case suggests that almonertinib has the potential to overcome resistance to osimertinib associated with the L718Q mutation and that NGS can be used as an effective method for EGFR mutation analysis. It may be very meaningful that NGS detection accompanies systemic treatment of advanced NSCLC patients or is used for monitoring NSCLC patients postoperatively. As more evidence accumulates, NGS accompanying monitoring may become a powerful clinical diagnosis and treatment tool.

\section{DATA AVAILABILITY STATEMENT}

The original contributions presented in the study are included in the article/Supplementary Material, further inquiries can be directed to the corresponding author.

\section{ETHICS STATEMENT}

The studies involving human participants were reviewed and approved by The Ethics Committee of the second affiliated hospital of Zunyi medical university. The patients/participants provided their written informed consent to participate in this study.

\section{AUTHOR CONTRIBUTIONS}

HZ, GS, and LS put forward the content of the paper. HZ wrote the manuscript. XT, LS, CG, DH, Jing Wang, XS, and HZ reviewed literature and clinical data. HZ prepared figures. HC helped to replenish the data. All authors read and approved the final manuscript.

Kinase Inhibitors for Advanced EGFR+ NSCLC. J. Thorac. Oncol. 16 (5), 740-763. doi:10.1016/j.jtho.2020.11.028

Remon, J., Steuer, C. E., Ramalingam, S. S., and Felip, E. (2018). Osimertinib and Other Third-Generation EGFR TKI in EGFR-Mutant NSCLC Patients. Ann. Oncol. 29 (Suppl. 1_1), i20-i27. doi:10.1093/annonc/ mdx704

Shaikh, M., Shinde, Y., Pawara, R., Noolvi, M., Surana, S., Ahmad, I., et al. (2021). Emerging Approaches to Overcome Acquired Drug Resistance Obstacles to Osimertinib in Non-small-cell Lung Cancer. J. Med. Chem. doi:10.1021/ acs.jmedchem.1c00876

Starrett, J. H., Guernet, A. A., Cuomo, M. E., Poels, K. E., van Alderwerelt van Rosenburgh, I. K., Nagelberg, A., et al. (2020). Drug Sensitivity and Allele Specificity of First-Line Osimertinib Resistance EGFR Mutations. Cancer Res. 80 (10), 2017-2030. doi:10.1158/0008-5472.CAN-19-3819

Taniguchi, H., Yamada, T., Wang, R., Tanimura, K., Adachi, Y., Nishiyama, A., et al. (2019). AXL Confers Intrinsic Resistance to Osimertinib and Advances the Emergence of Tolerant Cells. Nat. Commun. 10 (1), 259. doi:10.1038/s41467018-08074-0

Uchibori, K., Inase, N., Araki, M., Kamada, M., Sato, S., Okuno, Y., et al. (2017). Brigatinib Combined with Anti-EGFR Antibody Overcomes Osimertinib Resistance in EGFR-Mutated Non-small-cell Lung Cancer. Nat. Commun. 8, 14768. doi:10.1038/ncomms 14768

Webb, B., and Sali, A. (2016). Comparative Protein Structure Modeling Using MODELLER. Curr. Protoc. Bioinformatics 54 (2 9 1), 5-237. doi:10.1002/cpbi.3 
Wu, S. G., and Shih, J. Y. (2018). Management of Acquired Resistance to EGFR TKI-Targeted Therapy in Advanced Non-small Cell Lung Cancer. Mol. Cancer 17 (1), 38. doi:10.1186/s12943-018-0777-1

Yang, J. C., Camidge, D. R., Yang, C. T., Zhou, J., Guo, R., Chiu, C. H., et al. (2020). Safety, Efficacy, and Pharmacokinetics of Almonertinib (HS-10296) in Pretreated Patients with EGFR-Mutated Advanced NSCLC: A Multicenter, Open-Label, Phase 1 Trial. J. Thorac. Oncol. 15 (12), 1907-1918. doi:10.1016/j.jtho.2020.09.001

Yang, Z., Yang, N., Ou, Q., Xiang, Y., Jiang, T., Wu, X., et al. (2018). Investigating Novel Resistance Mechanisms to Third-Generation EGFR Tyrosine Kinase Inhibitor Osimertinib in Non-small Cell Lung Cancer Patients. Clin. Cancer Res. 24 (13), 3097-3107. doi:10.1158/1078-0432.CCR-17-2310

Yosaatmadja, Y., Silva, S., Dickson, J. M., Patterson, A. V., Smaill, J. B., Flanagan, J. U., et al. (2015). Binding Mode of the Breakthrough Inhibitor AZD9291 to Epidermal Growth Factor Receptor Revealed. J. Struct. Biol. 192 (3), 539-544. doi:10.1016/j.jsb.2015.10.018

Zhang, Y., Zhang, Y., Niu, W., Ge, X., Huang, F., Pang, J., et al. (2021). Experimental Study of Almonertinib Crossing the Blood-Brain Barrier in EGFR-Mutant NSCLC Brain Metastasis and Spinal Cord Metastasis Models. Front. Pharmacol. 12, 750031. doi:10.3389/fphar.2021.750031

Zhao, J., Zou, M., Lv, J., Han, Y., Wang, G., and Wang, G. (2018). Effective Treatment of Pulmonary Adenocarcinoma Harboring Triple EGFR Mutations of L858R, T790M, and Cis-C797s by Osimertinib, Bevacizumab, and Brigatinib Combination Therapy: a Case Report. Onco Targets Ther. 11, 5545-5550. doi:10.2147/OTT.S170358
Zhou, C., Xie, L., Liu, W., Zhang, L., Zhou, S., Wang, L., et al. (2021). Absorption, Metabolism, Excretion, and Safety of [14C]almonertinib in Healthy Chinese Subjects. Ann. Transl Med. 9 (10), 867. doi:10.21037/atm-21-1606

Conflict of Interest: Author HZ, CG, JW, XS, DH are employed by the company 3D Medicines Inc.

The remaining authors declare that the research was conducted in the absence of any commercial or financial relationships that could be construed as a potential conflict of interest.

Publisher's Note: All claims expressed in this article are solely those of the authors and do not necessarily represent those of their affiliated organizations, or those of the publisher, the editors and the reviewers. Any product that may be evaluated in this article, or claim that may be made by its manufacturer, is not guaranteed or endorsed by the publisher.

Copyright (c) 2021 Shen, Shi, Tian, Huang, Chen, Gao, Shen and Zhang. This is an open-access article distributed under the terms of the Creative Commons Attribution License (CC BY). The use, distribution or reproduction in other forums is permitted, provided the original author(s) and the copyright owner(s) are credited and that the original publication in this journal is cited, in accordance with accepted academic practice. No use, distribution or reproduction is permitted which does not comply with these terms. 